\title{
Vorwort zur 104. Auflage
}

1894 erschienen erstmals die „Logarithmischen Rechentafeln für Chemiker“. Damit kann der Küster/Thiel mit der vorliegenden Neuauflage auf eine fast 100jährige Tradition zurückblicken. Die wachsenden Anforderungen an die chemische und physikalisch-chemische Analytik - besonders die Entwicklungen im Umweltschutz, der Arbeitssicherheit, der Qualitätskontrolle und Prozeßsicherung - sind und bleiben eine ständige Herausforderung an Verlag und Autor, den Küster/Thiel aktuell zu halten, damit dem Benutzer auch in der Zukunft das passende Rüstzeug zur Auswertung und kritischen Beurteilung seiner Analysenergebnisse zur Verfügung steht.

Ein wichtiger Trend ist die immer schnellere, umfassendere und präziser werdende Erstellung von Analysendaten. Die moderne Mikroelektronik hat diesen Trend durch die enorme Vereinfachung von Auswertevorgängen drastisch beschleunigt. Die Gefahr, daß die mathematischen Zusammenhänge dabei verlorengehen und Ergebnisse unkritisch interpretiert und verarbeitet werden, ist groß. Diesem Sachverhalt wurde durch die Aufnahme des Kapitels 6 Statistische Auswertung von Versuchs- und Analysendaten Rechnung getragen. Die wichtigen Auswerte- und Kalibrierverfahren werden nebst statistischen Prüfverfahren anhand von Beispielen erläutert und sollen den Benutzer in die Lage versetzen, seine analytischen Arbeitsvorgänge kritisch zu bewerten.

Neu aufgenommen wurde das Kapitel 3 Spektroskopische Methoden zur Strukturaufklärung chemischer Verbindungen. Die Vereinfachung der Gerätetechnologie und des „Handlings“ haben dazu geführt, daß eine Reihe spektroskopischer Methoden vielfältigen Eingang in Synthese-, Betriebs- und Kontrollabors gefunden haben. Dem Studenten oder dem interessierten Praktiker sollen die vorliegenden Tabellen die Möglichkeit geben, Interpretationen von Spektren nachzuvollziehen bzw. eigene Ideen zur Produktidentifikation und Strukturaufklärung zu entwickeln. Die vielfältig vorhandene Spezialliteratur in Form von Spektrensammlungen soll damit nicht ersetzt werden.

Mein Dank gilt wiederum allen kritischen und aufmerksamen Lesern, die zur Gestaltung dieser Auflage beigetragen haben. Besonders hervorgehoben sei Herr Professor Dr. Gerhard Schulze (Technische Universität Berlin) für die wissenschaftliche Redaktion und für seine Beiträge, insbesondere zu Kapitel 6, sowie Herr Dr. Siegfried Noack (Bundesanstalt für Materialforschung und -prüfung, Berlin) ebenfalls für seine Anregungen und für seinen Beitrag zu Kapitel 6. Danken möchte ich auch Herrn Dipl.-Ing. D. Erxleben (Deutsches Institut für Normung e. V.) für seine Hinweise zur Manuskriptgestaltung. Mein Dank

gilt ferner meiner Frau Dr. Ursula Ruland für die intensive Unterstützung bei der Manuskripterstellung sowie dem Verlag Walter de Gruyter für die konstruktive Zusammenarbeit.

Altenbach, März 1993

Alfred Ruland 